\title{
El Compromiso Estudiantil en Educación Superior como Agencia Compleja
}

\author{
Juan E. Aspeé ${ }^{(1,2) \star}$, José A. González ${ }^{(3)}$ y Eduardo A. Cavieres-Fernández ${ }^{(3)}$ \\ (1) Pontificia Universidad Católica de Valparaíso, Avenida Brasil 2950, Valparaíso, Chile \\ (e-mail: juan.elias.aspee@gmail.com) \\ (2) Universidad Técnica Federico Santa María, Avenida España 1680, Valparaíso, Chile \\ (3) Universidad de Playa Ancha de Ciencias de la Educación, Avenida Playa Ancha 850, Valparaíso, Chile \\ (e-mail: jgonzalez@upla.cl; ecavieres-cea@upla.cl)
}

* Autor a quien debe ser dirigida la correspondencia

Recibido Dic. 15, 2017; Aceptado Feb. 21, 2018; Versión final Mar. 15, 2018, Publicado Ago. 2018

\section{Resumen}

Esta investigación analiza la expresión de grados y orientaciones del compromiso estudiantil, entendiendo que dicho fenómeno no se limita a las acciones académicas. Esto se logra mediante una aproximación cuantitativa, generando un índice del compromiso evidenciado entre estudiantes de una universidad regional de Chile (muestra de 412 estudiantes). Los resultados indican que la mayor parte de los estudiantes presentan un compromiso orientado a lo académico (en niveles medios y altos), pero que no se reduce a éste. También se detectó la emergencia del compromiso estudiantil complejo, aquel que no puede ser asociado a una sola orientación. Esto diversifica el concepto de compromiso estudiantil, más allá de lo exclusivamente académico, hacia su comprensión como una agencia compleja.

Palabras clave: compromiso estudiantil; educación superior; teoría del compromiso; compromiso complejo

\section{Student Engagement in Higher Education as a Complex Agency}

\begin{abstract}
The present research analyzes the degrees and orientations of student engagement, understood as a phenomenon that is not limited to academic actions. This is done through a quantitative approach that provides an index of clear commitment among students from a regional university in Chile (sample of 412 students). The results indicate that most of the students present an academic-oriented engagement (at medium and high levels), but not reducible to this. It was also observed the emergence of a complex student engagement, that which cannot be associated to a single orientation. This diversifies the concept of student engagement, beyond the exclusively academic aspect, towards its understanding as a complex agency.
\end{abstract}




\section{INTRODUCCIÓN}

A nivel mundial, las universidades están implementando procesos para asegurar que la formación que entregan se ajuste a estándares de calidad. Sin embargo, estos procesos han obviado la multidimensionalidad de este constructo (Krause, 2012), con escasa atención a las experiencias de los estudiantes (Coates, 2005). A raíz de ello, ha ido emergiendo el interés por investigar la perspectiva de los estudiantes, para captar desde ellos los aspectos que las universidades deberían mejorar. Dentro de estas investigaciones, sobresalen aquellas sobre el compromiso estudiantil, el que se ha considerado como un valor añadido que conduce hacia el aprendizaje y, por tanto, contribuye a los sistemas de aseguramiento de la calidad con información relevante acerca de los estudiantes y su experiencia formativa (Pascarella et al., 2010).

La mayoría de las investigaciones sobre el compromiso estudiantil se han desarrollado fundamentalmente en países anglosajones, iniciadas con el fin de comprender de mejor manera la asociación que existe entre este fenómeno y la permanencia de los estudiantes en sus estudios superiores; y el efecto que tiene sobre su integración social y académica en la universidad. Adicionalmente, estos estudios también están sirviendo para analizar los actuales procesos de mejoramiento de la educación superior en países como EE.UU. En la medida que procesos similares también están siendo implementados en Chile, y en general en Latinoamérica, las investigaciones sobre el compromiso estudiantil adquieren pertinencia para esta región del continente, especialmente ante la carencia de estudios en el área (Pineda-Báez et al. 2014).

En consecuencia, entendemos el compromiso estudiantil como el conjunto de acciones que realizan los estudiantes para alcanzar su éxito académico (LaNasa et al., 2009), pero no limitadas al aula. De esta manera, comprendemos el compromiso estudiantil como un fenómeno polisémico que revela la agencia de los estudiantes a través de la cual se involucran durante sus estudios con acciones que, incluso, no están consideradas dentro de los estándares de calidad definidos por las universidades (Kahn, 2014, 2017). Estas acciones pueden evidenciar un compromiso que destaca las cuestiones éticas y políticas de este fenómeno (McMahon y Portelli, 2004, 2012; Zepke y Leach, 2010). Así, por ejemplo en Chile, los movimientos de protestas estudiantiles, que buscan oponerse a la mercantilización de la educación superior y sus consecuencias en calidad y equidad (Bellei et al., 2014), pueden ser considerados parte del fenómeno del compromiso estudiantil, ya que los estudiantes podrían estar concibiendo su proceso formativo como parte de la transformación de la sociedad; esto en un contexto altamente masivo y privatizado de la educación superior. Esta perspectiva social, ha sido poco reflejada en las investigaciones en el área (Zepke, 2015, 2017), las que tienden a concentrarse en los aspectos más utilitarios del compromiso estudiantil, generalmente aludiendo a su componente académico. Por tanto, este estudio se focaliza en el compromiso estudiantil de 412 estudiantes de una universidad pública y regional de Chile, desde una perspectiva que enfatiza la agencia de los mismos, por la cual asumen acciones que constituyen distintas orientaciones del compromiso, integrando aspectos personales y ciudadanos, de manera de avanzar hacia una concepción más compleja del compromiso estudiantil.

En general, las perceptivas analíticas dominantes en el estudio del compromiso estudiantil se concentran en el comportamiento de los estudiantes y su proceso psicosocial, en las prácticas institucionales y en los contextos sociopolíticos en el cual se produce (Kahu, 2013). Asimismo, distintos estudios reconocen una variedad de factores que inciden en este compromiso, como los personales o de origen, y que están referidos al entorno familiar y la situación socioeconómica. Por ejemplo, junto al efecto adicional que produce la educación sobre el compromiso ciudadano de los estudiantes, los hijos de profesionales y/o alumnos con mayor recorrido laboral, tienen mayor tendencia a participar en actividades cívicas (Egerton, 2002). Del mismo modo, estudiantes con una experiencia institucional diversa, desarrollan un compromiso estudiantil menos ligado a requerimientos académicos tradicionales, sino que vinculado a otras experiencias sociales que igualmente redundan en el éxito con sus estudios (Lester et al., 2013). A su vez, otras investigaciones señalan que tanto el origen étnico/racial como el sexo, afectan el compromiso estudiantil (Chen et al., 2014). No obstante, algunos estudios han relativizado el efecto de estas características de entrada de los estudiantes, tal como ocurre con Jackling y Natoli (2011), quienes establecen que el sexo, el nivel socioeconómico, junto con el primer año de estudio, no son relevantes para determinar el compromiso de los alumnos. Consecuentemente, se requieren nuevos análisis para determinar de mejor manera este punto; aspecto que consideramos en la presente investigación.

El contexto educativo es otro factor que afecta el compromiso estudiantil (Zepke, 2014; Kahu y Nelson, 2017). Por un lado, el alcance de este compromiso se enmarca dentro de las normativas institucionales, como lo señala Carey (2016), las que pueden ampliar o limitar los espacios de participación estudiantil. Por otro lado, el compromiso de los estudiantes está influenciado por las pautas transaccionales de la cultura educativa de la que se forma parte y por las experiencias educativas que pudiesen ofrecer las universidades para favorecer, por ejemplo, la integración social (Brint et al., 2008). En este sentido, el compromiso estudiantil permite escudriñar el éxito académico en la universidad desde la perspectiva de los propios estudiantes y desde los 
efectos positivos que tiene sobre ellos. Este compromiso vuelve más probable la decisión de continuar con sus estudios, por medio de la integración académica y social que el mismo compromiso fomenta (Díaz, 2008).

No obstante la diversidad de variables y perspectivas que se han usado para el estudio del compromiso estudiantil, la mayoría de las investigaciones en el área han tendido a focalizarse en estándares ligados fundamentalmente al éxito académico, resaltando, por ende, los aspectos académicos del compromiso estudiantil y poniendo menos atención a la agencia de los estudiantes desde la cual asumen su formación en los entornos educativos (Kahn, 2014, 2017). En este sentido, concordamos que el compromiso estudiantil, como agencia, implica la capacidad reflexiva del alumno para actuar ante la incertidumbre, responsabilizándose de un proyecto de aprendizaje y con respuestas deliberativas que pueden exceder los márgenes establecidos por una exigencia académica formal (Kahn, 2014). Sin embargo, esto no significa que el compromiso estudiantil deba entenderse como una intervención de los estudiantes que está ajena a su rol académico. Más bien, representa el involucramiento del estudiante en su proceso formativo y las consecuencias individuales y sociales de tal, que considera tanto la responsabilidad con los estudios, como también la participación en otras actividades de su vida personal y social (Kuh et al., 2008; McMahon y Portelli, 2004, 2012). De esta manera, señalamos que el compromiso estudiantil es un fenómeno con una diversidad de orientaciones concretas, constituidas por una serie de acciones reflexivas dirigidas tanto al éxito académico, al desarrollo personal-integral de los estudiantes o al desarrollo ciudadano; ámbitos que, a su vez, se interrelacionan en los estudiantes. Entender al compromiso estudiantil considerando estas tres orientaciones permite avanzar hacia una comprensión más compleja de este fenómeno.

Consecuentemente, entendemos por una orientación académica del compromiso estudiantil las acciones del estudiante que se alinean esencialmente con el aprendizaje, derivando en logro académico (Jelas et al., 2016). En esta orientación, todos los esfuerzos se relacionan con los aprendizajes que los estudiantes desarrollan a partir de las actividades que realizan en el aula. Precisamente, en este alcance, un estudiante está dispuesto a invertir tiempo y esfuerzo para conseguir las metas académicas, de la mano con el nivel de calificaciones alcanzadas (Carini et al., 2006). Hacia dicha meta resulta crucial la intervención de los profesores, factor determinante en el aprendizaje efectivo (Valenzuela et al., 2016), quienes deben organizar sus prácticas pedagógicas para favorecer dicho compromiso (Wimpenny y Savin-Baden, 2013).

Por su parte, una orientación al desarrollo personal-integral del compromiso estudiantil contiene aquellas acciones que si bien están encaminadas al logro académico, fortalecen igualmente otras áreas de los estudiantes. En este sentido, el éxito académico no sólo depende de un esfuerzo y conducta individual, sino que involucra, entre otros aspectos, el resultado de trabajar con los demás (Zepke y Leach, 2010). Dentro de esta área, se incluyen acciones referidas a las interacciones que los estudiantes establecen con sus pares (Hu y McCormick, 2012), a la ejecución de actividades extra-programáticas o fuera del aula, pero complementarias a la formación, que ayudan al bienestar de los estudiantes (Krause y Coates, 2008), y a la diversidad en la interacción cultural y religiosa (Denson y Chang, 2008).

En tanto, una orientación hacia el desarrollo ciudadano del compromiso estudiantil, se conforma por aquellas acciones que buscan generar un ciudadano activo. Así, esta orientación constituye una experiencia de aprendizaje con beneficios sociales de largo plazo, tales como una mejor ciudadanía y una mejor sociedad. En esta orientación, el centro no es el estudiante como ente aislado, sino que es relevante su vínculo con las comunidades o contextos sociales y políticos más amplios (Jacoby, 2009). Por ende, incluye actividades como el mantenerse informado del acontecer nacional o internacional; participar en actividades deliberativas; ser miembros de asociaciones políticas o movimientos sociales; formar parte de grupos de voluntariado o de acción social; o participar en protestas públicas (Beaumont, Colby y Torney-Purta, 2006). Estas actividades favorecen el desarrollo de un pensamiento crítico respecto de lo que ocurre en la sociedad, vinculando los estudios con el mejoramiento del sistema político y democrático (McMahon y Portelli, 2004, 2012).

Por consiguiente, y situados en Chile, el presente escrito pretende contribuir al estudio de este compromiso, mostrando los resultados de una investigación de tipo cuantitativa, que recoge las orientaciones académicas, personales-integrales y ciudadanas del compromiso estudiantil, de los alumnos de una universidad regional perteneciente al Consejo de Rectores de Universidades de Chile (CRUCH). A esta razón, conviene señalar que, si bien en Chile existen investigaciones centradas en medir a los estudiantes de educación superior en relación al engagement académico, concepto asociado a estados mentales positivos a la conducta curricular, su devenir teórico y metodológico se aleja de la perspectiva sobre el compromiso estudiantil, con sus implicancias educativas y políticas aquí plasmada. Por lo demás, el mismo fenómeno del compromiso estudiantil ha sido poco estudiado en Latinoamérica, siendo el trabajo más relevante del área el de PinedaBaez et al. (2014), en Colombia, que también recalca este vacío teórico en la región. De este modo, esta investigación es original al integrar en un mismo análisis las orientaciones académicas, personales-integrales y ciudadanas del compromiso estudiantil en busca de ampliar su concepción. En tal sentido, se recalca la escasez de estudios que ponderen el fenómeno del compromiso estudiantil con la perspectiva aquí planteada, 
no tan sólo en la literatura latinoamericana, sino que incluso en la anglosajona (Zepke, 2014). En Chile es un momento favorable para este estudio, considerando que los estudiantes parecieran haber aumentado su orientación ciudadana del compromiso.

Por tanto, del conjunto de conceptos revisados, nos proponemos los siguientes objetivos: Objetivo 1: Identificar los grados de compromiso estudiantil expresados por los estudiantes; Objetivo 2: Identificar las orientaciones del compromiso estudiantil expresados por los estudiantes; y Objetivo 3: Indagar la relación entre el compromiso estudiantil y factores sociodemográficos y factores académicos. A su vez, a partir de estos objetivos sustentamos las siguientes hipótesis de trabajo (a un 0,05 de significancia): $H_{1}$ : Los alumnos expresan diferencias significativas entre grados de compromiso estudiantil; $H_{2}$ : Los alumnos expresan diferencias significativas entre las orientaciones del compromiso estudiantil; $H_{3}$ : La orientación académica del compromiso estudiantil predomina sobre la orientación personal-integral, la que a su vez predomina sobre la orientación ciudadana del compromiso estudiantil; $H_{4}$ : El grado y orientación del compromiso estudiantil se asocia significativamente con factores socio-demográficos; y $H_{5}$ : El grado y orientación del compromiso estudiantil se asocia significativamente con factores académicos.

\section{METODOLOGÍA}

En esta sección se describen aquellos elementos metodológicos que se utilizaron para llevar a cabo la investigación, y que permiten que este estudio pueda ser replicado, si así se desea, o bien, pueda ser revisado en términos procedimentales.

\section{Tipo y alcance de la investigación}

Investigación cuantitativa, transversal con alcance correlacional-explicativo.

\section{Universo y muestra}

El universo de estudio corresponde a todos los estudiantes de pregrado de una universidad regional de Chile, vigentes al primer semestre de 2017. La Tabla 1 presenta los elementos muestrales (seleccionados aleatoriamente), en función de la fórmula de Namakforoosh (2005), proporcionalizada según facultad y año de ingreso. Con un nivel de confianza del $95 \%$ y error de estimación del $\pm 5 \%$.

Tabla 1: Frecuencias de muestra, por facultad y año de ingreso a la institución

\begin{tabular}{|c|c|c|c|c|c|c|}
\hline \multirow[b]{2}{*}{ Facultad } & \multicolumn{5}{|c|}{ Año de ingreso a la institución } & \multirow[b]{2}{*}{ Total } \\
\hline & $\begin{array}{c}2012 \\
\text { (hacia atrás) }\end{array}$ & 2013 & 2014 & 2015 & 2016 & \\
\hline Facultad de Arte & 10 & 10 & 8 & 11 & 11 & 50 \\
\hline Facultad de Ciencias de la Actividad Física y el Deporte & 3 & 7 & 7 & 9 & 8 & 34 \\
\hline Facultad de Ciencias de la Educación & 2 & 10 & 9 & 7 & 11 & 39 \\
\hline Facultad de Ciencias de la Salud & 14 & 12 & 19 & 33 & 25 & 103 \\
\hline Facultad de Ciencias Naturales y Exactas & 12 & 3 & 5 & 4 & 10 & 34 \\
\hline Facultad de Ciencias Sociales & 7 & 9 & 6 & 11 & 14 & 47 \\
\hline Facultad de Humanidades & 14 & 17 & 12 & 10 & 20 & 73 \\
\hline Facultad de Ingeniería & 17 & 4 & 3 & 6 & 2 & 32 \\
\hline Total & 79 & 72 & 69 & 91 & 101 & 412 \\
\hline
\end{tabular}

La institución de educación superior elegida es una universidad estatal y regional que imparte diversos programas de estudio, que incluyen la mayor parte de las áreas del conocimiento (salud, ingenierías, ciencias sociales, humanidades, etc.). Por lo cual, su diversidad en términos de culturas disciplinarias enriquece los resultados. De la misma manera la universidad elegida es de acceso masivo, lo que garantiza la representación de una diversidad de condiciones de origen de los estudiantes, lo cual es atingente a los objetivos e hipótesis planteadas.

Considerando el nivel de confianza del $95 \%$ y error de estimación del $\pm 5 \%$ precedentemente planteados, la muestra alcanzó los 412 estudiantes. Así, cada elemento de la muestra representó a 11,3 elementos del universo estudiado (Factor de expansión = 4660/412). De estos el $72 \%$ son mujeres, mientras que el $28 \%$ son 
hombres. El 74,5\% de los estudiantes de la muestra provienen de hogares que están dentro del $60 \%$ de la población del país de menores ingresos económicos, mientras que el 14,3\% proviene del $40 \%$ de mayores recursos económicos. El 38\% de los alumnos de la muestra proviene de hogares donde el máximo nivel educativo alcanzado por los padres (o personas a cargo) fue la enseñanza media completa. Por otro lado, el $26 \%$ de los estudiantes de la muestra trabajó antes de ingresar a la educación superior, y lo continúa haciendo, mientras que el $32 \%$ no posee experiencia laboral previa, ni actual.

\section{Instrumento de recolección de datos}

Se utilizó un cuestionario estructurado-cerrado, auto-elaborado, tomando como referencia de contenido algunas preguntas incorporadas en el National Survey of Student Engagement (NSSE), que ha sido el instrumento más utilizado en el área y con mayor nivel de validación. Las preguntas fueron tomadas tanto del instrumento principal, como del modelo complementario para medir compromiso ciudadano (National Survey of Student Engagement, s. f. a, s. f. b). El cuestionario que desarrollamos contiene 30 ítems, distribuidos en 3 dimensiones, más una sección de antecedentes del estudiante informante, donde se incluyen sus calificaciones obtenidas en el último semestre, utilizadas como medida operativa del rendimiento educativo como reflejo del éxito educativo más amplio. Las dimensiones del instrumento se refieren exactamente a las conductas de las orientaciones del compromiso estudiantil señaladas en la introducción.

Definido operacionalmente el compromiso estudiantil como la frecuencia con la que un estudiante realiza determinadas acciones durante un semestre, el cuestionario se contestó en 5 escalas de intensidad por cada ítem, que dan cuenta del grado de involucramiento en su proceso formativo y que incluyen actividades orientadas al éxito académico, al desarrollo personal-integral y al desarrollo ciudadano (las 3 dimensiones indicadas).

Previo a la aplicación de este instrumento, a fines del año 2016 y principios del 2017 se realizó un estudio de calibración (de contenido, de consistencia y de contextualización) a partir de 51 ítems originales. Así, con una muestra de 108 casos, se llegó a un instrumento que presentó indicadores de fiabilidad elevados con un coeficiente alfa de Cronbach de 0,877 standardized alpha y de 0,875 alpha reliability. La validez del instrumento se determinó mediante análisis factorial exploratorio que permitió encontrar 11 factores de compromiso, distribuidos en forma independiente en cada una de las dimensiones del instrumento. Dicha validez fue ratificada por el uso de la validez gamma $(\Gamma)$ definida por González, Viveros y Carvajal (2016), cuyo indicador final alcanzó un coeficiente de 0,881 , que sigue la misma interpretación del alfa de Cronbach. Lo mismo ocurrió con la comprobación de validez de constructo mediante la utilización de hipótesis de correlación.

\section{Técnicas y proceso de análisis de datos}

El análisis de datos se realizó de manera incremental, en el siguiente sentido: 1) Análisis de resúmenes estadísticos; 2) Asociación entre categorías, mediante prueba Chi-cuadrado ( $\left.X^{2}\right)$; 3) Comparación de medidas de localización, con análisis previo de homocedasticidad y normalidad para aplicar pruebas paramétricas [ANOVA de un factor $(F)$ y T test para grupos independientes $(t)$ ], o no paramétricas [Estadístico $\mathrm{H}$ o KruskalWallis chi-cuadrado $(H)$, y Prueba $U$ de Mann-Whitney o suma de rangos de Wilcoxon $(W)$ ]; 4) Correlación de Spearman (bilateral); y 5) Modelo lineal generalizado con función de enlace la identidad.

\section{Índice de compromiso estudiantil}

Los análisis descritos en el apartado anterior, se centran en los puntajes obtenidos en el instrumento de recolección de datos por cada alumno, que se traduce en un índice que describe tanto el grado de compromiso estudiantil, como su orientación predominante, a saber: Sean los niveles principales Nivel A (dimensión académica); Nivel B (dimensión personal-integral); y Nivel C (dimensión ciudadana). Sean las siguientes formulas los puntajes máximos a obtener en cada uno de los niveles principales, donde $N_{A}, N_{B}$ y $N_{C}$, expresan respectivamente el puntaje máximo posible de obtener en Nivel A, Nivel B, y Nivel C:

$$
\begin{aligned}
& P_{U}(A)=\frac{1}{N_{A}} \\
& P_{U}(B)=\frac{1}{N_{B}} \\
& P_{U}(C)=\frac{1}{N_{C}}
\end{aligned}
$$


Sean $\mathrm{n}_{\mathrm{A}}, \mathrm{n}_{\mathrm{B}}$ y $\mathrm{n}_{\mathrm{C}}$ los puntajes obtenidos por cada individuo en los niveles principales $\mathrm{A}, \mathrm{B}$ y $C$, respectivamente. Entonces, se definen las probabilidades de ocurrencia de cada nivel principal, A, B y C, como:

$$
\begin{aligned}
& P(A)=P_{U}(A) \cdot n_{A}=\frac{n_{A}}{N_{A}} \\
& P(B)=P_{U}(B) \cdot n_{B}=\frac{n_{B}}{N_{B}} \\
& P(C)=P_{U}(C) \cdot n_{C}=\frac{n_{C}}{N_{C}}
\end{aligned}
$$

Como A, B y C son disjuntos, sea el test total como:

$$
T=A \cup B \cup C
$$

Luego la probabilidad de acierto en el test total se define como:

$$
P(T)=\frac{\frac{n_{A}}{N_{A}}+\frac{n_{B}}{N_{B}}+\frac{n_{C}}{N_{C}}}{3}
$$

El nivel de logro para $T$, entendido como el grado de compromiso estudiantil evidenciado, puede ser distribuido en cinco secciones o niveles de logro:

Si $P(T) \in[0,0 ; 0,2[=$ el nivel de logro es muy bajo

Si $P(T) \in[0,2 ; 0,4[=$ el nivel de logro es bajo

Si $P(T) \in[0,4 ; 0,6[=$ el nivel de logro es medio

Si $\mathrm{P}(\mathrm{T}) \in[0,6 ; 0,8]=$ el nivel de logro es alto

Si $P(T) \in[0,8 ; 1,0]=$ el nivel de logro es muy alto

Para establecer cuál de los tres niveles es el principal que identifica al individuo, se utiliza el máximo entre $P(A), P(B)$ y $P(C)$, esto es:

$$
\operatorname{Max}\{P(A), P(B), P(C)\}=\text { orientación principal }
$$

Ahora bien, si existen a lo menos dos probabilidades de ocurrencia de cada nivel principal iguales y máximas, entonces no existirá una orientación predominante, sino que la denominaremos de compromiso complejo.

En adelante, se indicará $\mathrm{P}(\mathrm{T})$ como $\mathrm{PT}, \mathrm{P}(\mathrm{A})$ como $\mathrm{PA}, \mathrm{P}(\mathrm{B})$ como $\mathrm{PB}$ y $\mathrm{P}(\mathrm{C})$ como $\mathrm{PC}$.

\section{Variables analizadas}

Finalmente, las variables analizadas se dividen en las categorías descritas en la Tabla 2.

Tabla 2: Categorías de variables e hipótesis relacionadas

\begin{tabular}{|c|c|c|}
\hline \multirow{2}{*}{ Categoría } & Variables & $\begin{array}{c}\text { Hipótesis } \\
\text { relacionada }\end{array}$ \\
\hline \multirow{3}{*}{$\begin{array}{c}\text { Índice de } \\
\text { compromiso }\end{array}$} & PT & $H_{1}$ \\
\cline { 2 - 3 } & PA & $H_{2} ; H_{3}$ \\
\cline { 2 - 3 } & PB & \\
\hline
\end{tabular}


Tabla 2 (continuación)

\begin{tabular}{|c|c|c|}
\hline Categoría & Variables & $\begin{array}{l}\text { Hipótesis } \\
\text { relacionada }\end{array}$ \\
\hline \multirow{7}{*}{$\begin{array}{c}\text { Factores } \\
\text { sociodemográficos }\end{array}$} & Sexo $(S X)$ & \multirow{7}{*}{$H_{4}$} \\
\hline & Año de ingreso a la institución (All) & \\
\hline & Quintil de ingreso per cápita al que pertenece el estudiante (QIPC) & \\
\hline & $\begin{array}{l}\text { Dependencia administrativa del establecimiento de egreso de enseñanza } \\
\text { media (DAE) }\end{array}$ & \\
\hline & Nivel máximo de escolaridad alcanzado por padres o tutores (NME) & \\
\hline & Experiencia laboral previa al ingreso a la educación superior (ELP) & \\
\hline & Experiencia laboral actual -estudia y trabaja- (ELA) & \\
\hline \multirow{2}{*}{$\begin{array}{l}\text { Factores } \\
\text { académicos }\end{array}$} & Facultad de Estudios (FCD) & \multirow{2}{*}{$H_{5}$} \\
\hline & Media de calificaciones de último semestre informada por estudiante & \\
\hline
\end{tabular}

\section{RESULTADOS}

Los resultados dan sustento a $H_{1}$. Así, existen diferencias significativas entre los grados de compromiso estudiantil (ver Fig. 1), ratificado por la comparación de medianas, siendo la más favorable para el compromiso estudiantil muy alto, y más desfavorable para el compromiso estudiantil bajo $(H=349,7$; $p$-valor $<2,2 e-16$; $<0.05)$. De hecho, todas las comparaciones entre grados de compromiso presentan diferencias significativas (test de comparaciones múltiples Kruskal-Wallis, a 0,05). Este resultado refleja la consistencia del índice, cuyas categorías analíticas se determinan en función del mismo puntaje total obtenido. Es decir, son categorías excluyentes.

Los resultados también confirman $\mathrm{H}_{2}$, los que arrojan diferencias significativas entre las orientaciones de compromiso estudiantil (ver Fig. 2), al compararse sus medianas de puntaje total $(H=12,028$; $p$-valor 0,007 ; $<0,05)$. De hecho, la orientación académica es significativamente diferente de la orientación personal-integral del compromiso (análisis post Kruskal-Wallis, flexibilizando $\alpha$ un $8 \%$ ).

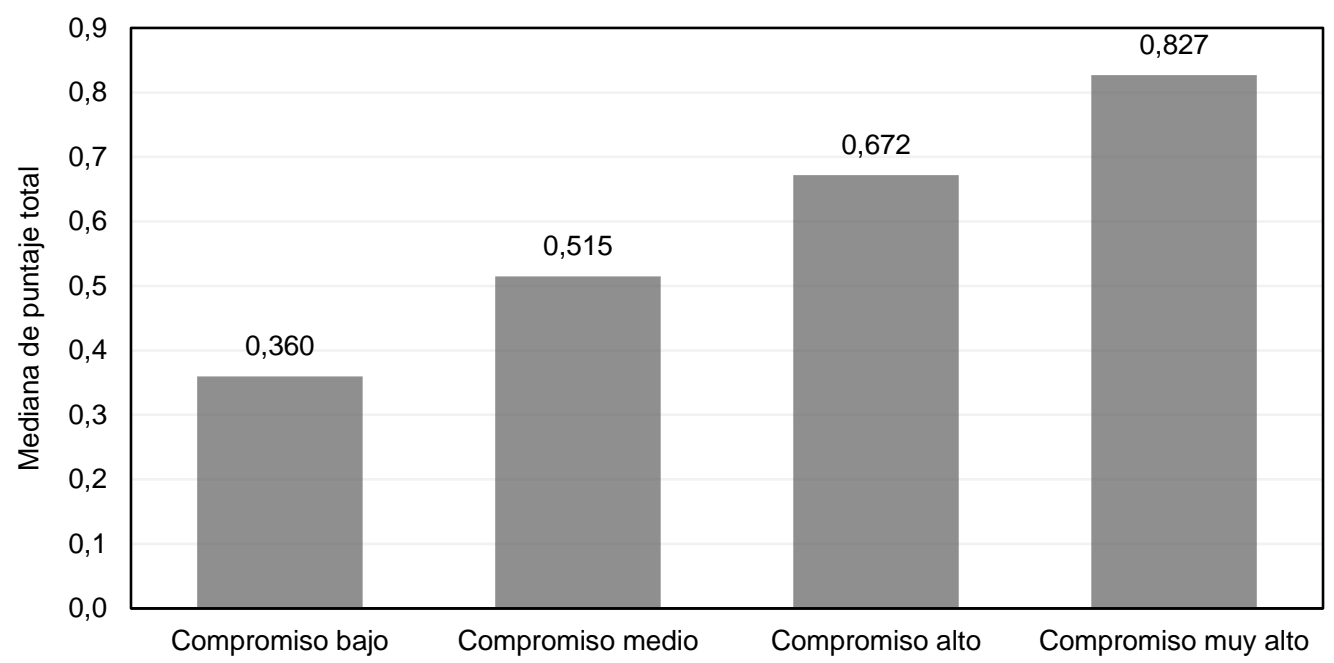

Fig. 1: Mediana de puntaje total, según grado de compromiso

Asimismo, se indica que existen diferencias significativas en los puntajes de PA y las orientaciones del compromiso estudiantil $(H=65,471$; p-valor 3,978 e-14; $<0,05)$. Diferencia perceptible entre alumnos con una orientación académica versus aquellos con una orientación ciudadana; y aquellos con una orientación personal-integral (test de comparaciones múltiples de Kruskal-Wallis, a 0,05). Lo mismo para el caso de los puntajes de PB y las orientaciones del compromiso $(H=63,013 ; p$-valor $1,334 \mathrm{e}-13 ;<0,05)$. Diferencia sensible entre alumnos con una orientación personal-integral y aquellos con una orientación académica; y con una orientación ciudadana del compromiso (test de comparaciones múltiples de Kruskal-Wallis, a 0,05). Y lo mismo para los puntajes de PC, según orientaciones del compromiso estudiantil $(H=78,919$; $p$-valor $<2$.2e$16 ;<0,05)$. Diferencias detectables para todos los pares de comparaciones de orientaciones de compromiso, excepto para la comparación entre la orientación compleja y la ciudadana, y entre la orientación compleja y la orientación personal-integral del compromiso (test de comparaciones múltiples de Kruskal-Wallis, a 0,05). 


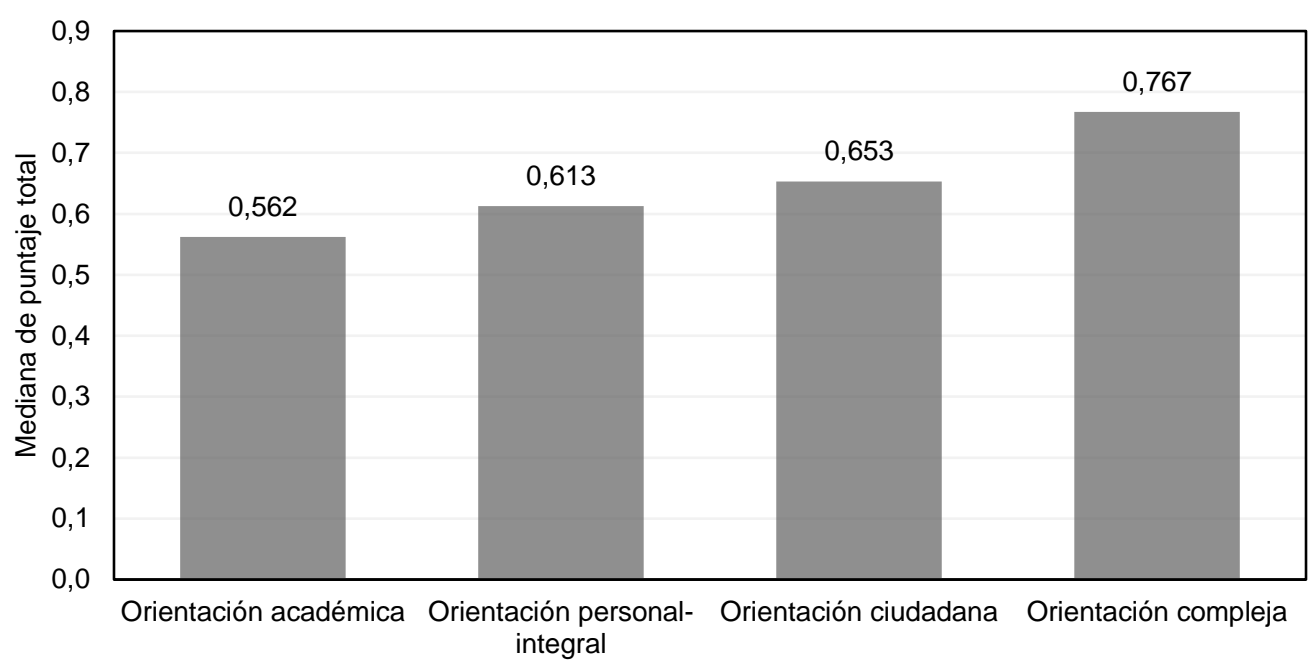

Fig. 2: Mediana de puntaje total, según orientación del compromiso

Con relación a $\mathrm{H}_{3}$, los resultados muestran que la orientación académica del compromiso predomina sobre la orientación personal-integral, la que a su vez predomina sobre la orientación ciudadana (Tabla 3). Los estudiantes muestran diferentes grados de compromiso estudiantil, con una mayoría de ellos orientados a lo académico, en grado medio. No obstante, casi un tercio de los estudiantes expresan tener otra orientación principal, incluyendo algunos que adscriben a la orientación que hemos denominado compleja. Además, se destaca el hecho de que no existe representación de la categoría de compromiso muy bajo.

Los resultados corroboran parcialmente $\mathrm{H}_{4}$, debido a que los grados de compromiso (Tabla 4), no presentan una asociación significativa respecto al quintil de ingreso por persona (QIPC). Sin embargo, existen diferencias significativas entre las medias del puntaje total, diferencias que se dan entre estudiantes del quintil 4 versus estudiantes del quintil 3 (test de comparaciones múltiples Tukey, flexibilizando un $5 \% \alpha$ ). Esta situación no se da con ninguna otra variable.

Tabla 3: Grado y orientación del compromiso estudiantil

\begin{tabular}{|l|c|c|c|c|c|}
\hline \multirow{2}{*}{ Grado de compromiso } & \multicolumn{4}{|c|}{ Orientación del compromiso } & \multirow{2}{*}{ Total } \\
\cline { 2 - 6 } & Académico & Personal-Integral & Ciudadano & Complejo \\
\hline Bajo & 28 & 5 & 8 & 1 & 42 \\
\hline Medio & 143 & 24 & 12 & 2 & 181 \\
\hline Alto & 100 & 35 & 26 & 3 & 164 \\
\hline Muy alto & 13 & 5 & 6 & 1 & 25 \\
\hline Total & 284 & 69 & 52 & 7 & 412 \\
\hline
\end{tabular}

Tampoco es posible establecer relación entre las orientaciones de compromiso estudiantil y el quintil de ingreso per cápita de los estudiantes (Tabla 5), aunque las conductas asociadas a lo personal-integral, contenidas en puntaje PB, están levemente influenciadas por el paso de un quintil a otro. Así, se observan diferencias entre alumnos del quintil 4 y del quintil 3, a favor del quintil 4 (test de comparaciones múltiples Kruskal-Wallis, flexibilizando un $2 \%$ a). Del mismo modo, no existe relación entre la orientación del compromiso y el máximo nivel educativo alcanzado por padres o tutores. Sin embargo, respecto de las conductas asociadas al compromiso de orientación personal-integral, existen diferencias significativas a favor de alumnos provenientes de hogares con mayor nivel educativo.

Estas diferencias se detectan en el puntaje PB entre estudiantes con padres cuyo nivel máximo de escolaridad es la enseñanza media completa versus alumnos con padres cuyo nivel máximo de escolaridad es la enseñanza superior completa, a favor de estos últimos (test de comparaciones múltiples Kruskal-Wallis, flexibilizando un $8 \% \alpha$ ). Esta situación que no se da con ninguna otra variable (ver Tabla 5). Es pertinente señalar que para el uso de técnicas de análisis de datos contenidas en la Tabla 4 y la Tabla 5, se analizó homocedasticidad y normalidad para el uso de pruebas paramétricas y pruebas no paramétricas, según necesidad. Asimismo, el nivel de significancia para interpretar los resultados fue de un a 0,05. 
Tabla 4: Resultados entre grados de compromiso y factores asociados

\begin{tabular}{|c|c|c|}
\hline Variable & $\begin{array}{c}\text { Asociación } X^{2} \text { con grados de compromiso }(\alpha \\
0,05)\end{array}$ & $\begin{array}{c}\text { Diferencias entre medidas de localización con } \\
P T(\alpha 0,05)\end{array}$ \\
\hline FCD & $X^{2}=34,76 ; p$-valor 0,030 & $H=17,133 ; p$-valor 0,017 \\
\hline SX & $X^{2}=1,2324 ; p$-valor 0,745 & $t=0,40859 ; p$-valor 0,683 \\
\hline All & $X^{2}=18,804 ; p$-valor 0,093 & $F=1,46 ; p$-valor 0,214 \\
\hline QIPC & $X^{2}=16,382 ; p$-valor 0,174 & $F=2,973 ; p$-valor 0,020 \\
\hline DAE & $X^{2}=4,9288 ; p$-valor 0,553 & $F=0,667 ; p$-valor 0,514 \\
\hline NME & $X^{2}=14,117 ; p$-valor 0,722 & $t=-1,5081 ; p$-valor 0,132 \\
\hline ELP & $X^{2}=5,9841 ; p$-valor 0,112 & $t=-1,441 ; p$-valor 0,151 \\
\hline ELA & $X^{2}=3,8036 ; p$-valor 0,284 & -valor 0,107 \\
\hline
\end{tabular}

Tabla 5: Resultados entre orientaciones del compromiso y factores asociados

\begin{tabular}{|c|c|c|c|c|}
\hline Variable & $\begin{array}{c}\text { Asociación } X^{2} \text { con } \\
\text { orientaciones del } \\
\text { compromiso }(\alpha \text { 0,05) }\end{array}$ & $\begin{array}{c}\text { Diferencias entre } \\
\text { medidas de localización } \\
\text { con PA ( } \alpha \text { 0,05) }\end{array}$ & $\begin{array}{c}\text { Diferencias entre } \\
\text { medidas de localización } \\
\text { con PB }(\alpha \text { 0,05) }\end{array}$ & $\begin{array}{c}\text { Diferencias entre } \\
\text { medidas de localización } \\
\text { con PC }(\alpha \text { 0,05) }\end{array}$ \\
\hline FCD & $X^{2}=38,182 ; p$-valor 0,012 & $H=11,223 ; p$-valor 0,129 & $H=25,528 ; p$-valor 0,001 & $H=19,77 ; p$-valor 0,006 \\
\hline SX & $X^{2}=4,6624 ; p$-valor 0,198 & $W=16471 ; p$-valor 0,634 & $\begin{array}{c}W=18297 ; p \text {-valor } \\
0,225\end{array}$ & $W=17480 ; p$-valor 0,648 \\
\hline All & $X^{2}=18,798 ; p$-valor 0,094 & $H=3,2255 ; p$-valor 0,521 & $H=6,5713 ; p$-valor 0,160 & $H=3,1424 ; p$-valor 0,534 \\
\hline QIPC & $X^{2}=18,58 ; p$-valor 0,099 & $H=8,695 ; p$-valor 0,069 & $H=11,056 ; p$-valor 0,026 & $H=5,9717 ; p$-valor 0,201 \\
\hline DAE & $X^{2}=5,8921 ; p$-valor 0,435 & $H=1,3949 ; p$-valor 0,498 & $H=1,233 ; p$-valor 0,540 & $H=0,1995 ; p$-valor 0,905 \\
\hline NME & $X^{2}=20,507 ; p$-valor 0,305 & $H=7,0754 ; p$-valor 0,314 & $H=14,893 ; p$-valor 0,021 & $H=7,7909 ; p$-valor 0,254 \\
\hline ELP & $X^{2}=2,5793 ; p$-valor 0,461 & $W=19986 ; p$-valor 0,483 & $W=19388 ; p$-valor 0,230 & $W=18566 ; p$-valor \\
& $X^{2}=3,0125 ; p$-valor & $W=18704 ; p$-valor 0,342 & $W=18575 ; p$-valor 0,289 & $W=18165 ; p$-valor \\
\hline ELA & 0,390 & & & 0,158 \\
\hline
\end{tabular}

Los resultados sustentan $H_{5}$. En efecto, el grado de compromiso (Tabla 4) se asocia a la facultad de estudios, existiendo diferencias significativas entre las medianas de puntajes totales según facultades. Por ejemplo, los estudiantes de la Facultad de Ciencias de la Actividad Física y el Deporte muestran un mayor grado de compromiso que los de la Facultad de Ciencias de la Salud (test de comparaciones múltiples Kruskal-Wallis flexibilizado a un $1 \%$ sobre $\alpha$ ). Por su parte, y respecto de las orientaciones, existen diferencias significativas para los puntajes PB y PC relacionado a las facultades a las que pertenecen los estudiantes (Tabla 5). Las diferencias en el puntaje de PB son detectables entre alumnos de la Facultad de Ciencias Físicas y del Deporte versus alumnos de la Facultad de Ciencias Naturales y Exactas, de la Facultad de Ciencias Sociales, y de la Facultad de Ingeniería (test de comparaciones múltiples Kruskal-Wallis, a 0,05), a favor de los primeros. Las diferencias en el puntaje $\mathrm{C}$ son detectables entre la Facultad de Ciencias sociales y la Facultad de Ciencias de la Salud (test de comparaciones múltiples Kruskal-Wallis, flexibilizando $4 \%$ a), siendo favorables para los estudiantes de ciencias sociales.

Igualmente, existe una correlación positiva y significativa entre el promedio de calificaciones y el puntaje total obtenido para el compromiso estudiantil, así como entre la calificación promedio obtenida en el último semestre y el puntaje de PA (Tabla 6); siendo no significativa para los puntajes de PB y PC y promedio de notas. Por ende, se plantea un modelo explicativo tomando como única variable independiente el puntaje obtenido en PA.

De esta manera se tiene que:

$$
Y=\beta_{0}+\beta_{1} X_{1}
$$

Donde $Y$ es el promedio de calificaciones informada por estudiante, $\beta_{0}$ es el intercepto o valor de ajuste, $\beta_{1}$ es el efecto en promedio de calificaciones de puntaje PA, y $\mathrm{X}_{1}$ es la variable continua asociada al puntaje de PA.

Con base al $p$-valor $(0,008308 ;<0,05)$, el modelo que explica el rendimiento académico en función del puntaje obtenido en PA es significativo (ver Tabla 7). El porcentaje de la variabilidad explicado por PA es de un 1,5\%. 
Por ende, son las acciones relacionadas exclusivamente a la orientación académica del compromiso las que tienen un impacto relevante en el rendimiento; aunque las acciones de la orientación personal-integral y la dimensión ciudadana no lo afectan negativamente.

Tabla 6: Correlación de Spearman

\begin{tabular}{|l|l|c|}
\hline \multicolumn{1}{|c|}{ Relación: correlación de Spearman (bilateral) } & Rho & $p$-valor (a 0,05) \\
\hline Calificación promedio obtenida en último semestre y puntaje de PT & 0,105 & 0,033 \\
\hline Calificación promedio obtenida en último semestre y puntaje de PA & 0,139 & 0,005 \\
\hline Calificación promedio obtenida en último semestre y puntaje de PB & 0,078 & 0,113 \\
\hline Calificación promedio obtenida en último semestre y puntaje de PC & 0,058 & 0,241 \\
\hline
\end{tabular}

Tabla 7: Valores de modelo explicativo del rendimiento, con base a puntajes PA

\begin{tabular}{|c|c|c|}
\hline Parámetro & Estimación & $p$-valor $(\alpha 0,05)$ \\
\hline$\beta_{0}$ & 49,073 & $<2 \mathrm{e}-16$ \\
\hline$\beta_{1}$ & 5,482 & 0,008 \\
\hline
\end{tabular}

\section{DISCUSIÓN}

En este estudio nos hemos focalizado en el compromiso estudiantil, a partir de una muestra de 412 estudiantes chilenos, subrayando como perspectiva la agencia de los estudiantes por la cual asumen acciones que constituyen distintas orientaciones del compromiso. Para ello hemos medido los grados y las orientaciones del compromiso de estos estudiantes, así como la relación entre su compromiso y factores socio-demográficos y académicos.

De este modo, se acreditó que, si bien la mayor parte de los estudiantes presentan una orientación académica del compromiso en niveles medios y altos, es posible identificar la existencia de diferentes grados de compromiso estudiantil y de orientaciones. En particular, se estableció que las orientaciones del compromiso estudiantil son significativamente diferentes entre sí, con excepción del compromiso estudiantil complejo, que no detecta diferencias significativas con el compromiso de orientación personal-integral y ciudadano. En otras palabras, un alumno con mayores grados de desarrollo de la orientación personal-integral y/o ciudadana tiene más propensión a presentar un compromiso complejo, pues en la generalidad presentarían ya un compromiso académico medio o alto. Por ende, el compromiso académico aparece como el mínimo común denominador que caracteriza a todos los estudiantes analizados, pero que no se reduce a él. De hecho, hay alumnos que enfatizan otras orientaciones dentro de su compromiso, e incluso, algunos presentan un compromiso complejo al conjugar más de una orientación en su accionar. En este sentido, se da un sustento empírico a los planteamientos de Kahn $(2014,2017)$, que son fundamentales para este trabajo, y según los cuales, la agencia reflexiva de los estudiantes permite incorporar conductas académicas y no ortodoxamente académicas a su compromiso, que amplían el rango de su experiencia educativa. Esto ayuda a entender al compromiso estudiantil como un sólo fenómeno, pero con diferentes expresiones que varían de sujeto a sujeto.

Más aún, lo anterior da base para subrayar que las universidades deben promover un compromiso complejo, donde la orientación académica esté abierta a aprendizajes extra-programáticos que ayuden al bienestar de los estudiantes en lo personal y que deriven en la generación de ciudadanos activos. Esta evidencia apoya la indicación de Zepke $(2014,2017)$ de superar la comprensión reduccionista del compromiso estudiantil, mostrando que es posible la ocurrencia de varias orientaciones de este fenómeno, ya sea en el agregado de personas o incluso en un mismo estudiante. A su vez, da cuenta de que la orientación ciudadana del compromiso no está desconectada del logro académico (Zepke, 2015), sino que es parte de un conjunto complejo de acciones con las que está recíprocamente interrelacionado. Este hecho, al tiempo de enriquecer la formación del propio estudiante, posibilita un efecto performativo en la educación superior que dota al estudiante de una capacidad de transformación social en busca de una mejor sociedad (Bellei et al. 2014). Consecuentemente, los resultados permiten nuevas respuestas a las preguntas sobre el para qué del compromiso estudiantil y, por tanto, cuáles son los aspectos en los que los estudiantes también debieran comprometerse más allá de su éxito académico (McMahon y Portelli, 2004).

En general, los datos de la investigación entregaron evidencia en contra de una relación significativa entre los factores de entrada de los estudiantes y el compromiso estudiantil. Consecuentemente, los resultados refuerzan las deducciones de algunas investigaciones en las que ni el sexo ni la trayectoria educativa tienen 
una relación significativa en los grados de compromiso (Jackling y Natoli, 2011). Igualmente, y a diferencia de otras investigaciones en el área (Egerton, 2002), la investigación no arroja una relación estadística significativa entre el compromiso de los estudiantes y su trayectoria laboral, o entre nivel educativo (profesional) de los padres y dicho compromiso. No obstante, sí fue posible detectar en los datos algunas diferencias en función del origen socioeconómico de los estudiantes respecto al compromiso estudiantil; lo que impide descartar del todo la influencia de factores de entrada en dicho compromiso. Puesto que la expresión de los factores de entrada no es estandarizada o invariable (Lester et al. 2013), su escudriñamiento futuro debería incorporar nuevos instrumentos y muestras de participantes que reflejen la variabilidad con que estos factores interactúan con los contextos institucionales de educación superior en donde se desarrolla el compromiso estudiantil. Estos contextos educativos pudieran estar minimizando el efecto de las características de entrada, resaltando la influencia institucional en la expresión del compromiso. Así, por ejemplo, programas con régimen diurno de estudios, como los considerados en esta investigación, podrían de por si estar relegando la importancia de la experiencia laboral para el compromiso de los estudiantes, en comparación con programas de estudios vespertinos en donde la proporción de estudiantes con trabajos remunerados es significativamente mayor. Desde esa perspectiva, investigaciones a futuro deberían concentrarse en dar más luces respecto de la relación entre factores de origen y compromiso estudiantil, y complementando lo evidenciado en este trabajo, entender las acciones que pudieran realizar las universidades para considerar debidamente a dichos factores en busca de aumentar el compromiso de sus estudiantes.

Respecto a la asociación entre compromiso estudiantil y rendimiento académico, la investigación señala que es positiva, específicamente en relación a la orientación académica, lo que junto con confirmar otros estudios en el área (Carini et al. 2006; Pineda-Báez et al. 2014), otorga validez al instrumento e índice utilizados. Ello, por tanto, constriñe a las universidades a reforzar aquellas acciones que favorecen esta vinculación en favor del éxito académico de sus estudiantes. En ese sentido, en la medida en que los sistemas educativos tienden a vincular cada vez más los procesos de aprendizaje generados en los ciclos escolares básicos y secundarios con los de la educación superior, resulta importante complementar los resultados de este estudio con otros que han establecido la asociación entre el rendimiento previo al ingreso a la educación superior y el éxito educativo que el estudiante posteriormente alcanza en ella (Palominos et al., 2018). Como consecuencia, el instrumento aplicado en esta investigación y los hallazgos obtenidos, pueden servir para investigaciones específicas dentro del ámbito escolar acerca de cómo promover a ese nivel el compromiso de los estudiantes en vistas a su rendimiento académico, y cómo aquello puede redundar en el compromiso que los estudiantes desarrollan luego en sus estudios superiores.

No obstante todo lo anterior, el presente trabajo tampoco descarta la importancia de las conductas personalesintegrales y ciudadanas para el rendimiento académico, las que no generan efectos negativos sobre él. Por ende, se requiere poner atención a las acciones que las universidades realizan y podrían realizar para no desvincular lo académico con el progreso interpersonal, comunitario y ciudadano de los estudiantes (Beaumont et al. 2006), a fin de que la formación superior no pierda su componente social y su capacidad performativa anteriormente descrita. Considerando aquello, son relevantes algunos hallazgos obtenidos en este estudio. Así, el hecho que los estudiantes que forman parte de la Facultad de Ciencias Físicas y del Deporte presenten un mayor grado de compromiso estudiantil con una orientación predominantemente personal-integral, podría estar apuntando al efecto de la cultura institucional, aspecto que algunas investigaciones resaltan como elemental para promover grados y orientaciones específicas del compromiso estudiantil (Brint et al. 2008), o incluso a las acciones que dentro de dicha cultura están normadas (Carey, 2016). De esta manera, es lógico pensar que una facultad destinada a la formación de profesionales de la actividad física, presente niveles de compromiso personal-integral más sustantivos que otras unidades académicas. Mismo efecto puede detectarse en los estudiantes pertenecientes a la Facultad de Ciencias Sociales, quienes presentan una tendencia mayor a la orientación ciudadana del compromiso. Esto refuerza la noción de que los sistemas de aseguramiento de la calidad no pueden privilegiar estándares académicos, sin considerar los aportes que las mismas universidades ofrecen en otras áreas del desarrollo estudiantil (Coates, 2005). Consecuentemente, se abre la necesidad de nuevas investigaciones que analicen más en detalle las diferencias en las culturas del compromiso estudiantil que se podrían estar desarrollando en las diversas unidades o facultades de las universidades y en las cuales la reflexividad comportamental de los estudiantes, en vistas a su éxito académico, se va enmarcado en las mismas pautas transaccionales del contexto educativo del que forman parte.

Junto a las contribuciones y a las preguntas de investigación a futuro que ya hemos detallado, emergen otros aspectos a considerar. Así, se debe complementar esta investigación con la comparación distribucional del índice del compromiso estudiantil utilizado, más allá de la comparación de medidas de localización aquí ya presentadas. Esto permitiría caracterizar paramétricamente al compromiso estudiantil, a fin de entender su expresión en el propio universo de estudio. De igual forma, se sugiere aplicar el instrumento en otros escenarios corporativos e institucionales de educación superior, de manera que dicha diversidad pueda ir afinándolo. Ello permitirá continuar accediendo a nuevos datos que enriquezcan la perspectiva a que hemos 
avanzado y que ayuda a superar una concepción meramente academicista del compromiso. Estos estudios en el futuro podrán, por tanto, establecer con mayor claridad cómo las universidades, en sus respectivo contextos locales y nacionales -en muchos de los cuales se están implementando mecanismos de aseguramiento de la calidad- puedan facilitar una agencia de los estudiantes que esté abierta a un mayor espectro de actividades y orientada hacia comprensiones más complejas del compromiso estudiantil.

\section{CONCLUSIONES}

Los resultados y discusiones precedentes nos permiten estipular que: A) La expresión de grados y orientaciones del compromiso estudiantil debe ser entendida como un fenómeno complejo, no estático, resultado de la reflexividad de las acciones ejecutadas por los estudiantes, pero enmarcadas por las instituciones de las que forman parte que las promueven o limitan configurando su campo de acción; B) La expresión compleja del compromiso estudiantil, refuerza el hecho de que el compromiso va más allá de lo exclusivamente académico, incorporando acciones que, fuera del aula, igualmente aportan al proceso formativo. Ello no significa desvalorar lo académico, más bien subraya la importancia de los componentes personales-integrales y ciudadanos de este fenómeno, que tradicionalmente han sido relegados a un segundo plano por la preponderancia del rendimiento académico; y C) Las instituciones de educación superior deben promover mayores grados y diversificación del compromiso, considerando estrategias que se adapten y/o reconozcan tanto a las condiciones de origen de los estudiantes como las condiciones de contexto, centrados en la agencia de los mismos por la cual se involucran en su proceso formativo con acciones académicas, personales-integrales y/o ciudadanas, de maneras crecientemente complejas.

\section{AGRADECIMIENTOS}

Los autores agradecen al programa de Doctorado en Políticas y Gestión Educativa de la Universidad de Playa Ancha, Valparaíso Chile, desde donde se gestó esta investigación.

\section{REFERENCIAS}

Beaumont, E., T. Colby y J. Torney-Purta, Promoting Political Competence and Engagement in College Students: An Empirical Study, doi: 10.1080/15512160600840467, Journal of Political Science Education, 2(3), 249-270 (2006)

Bellei, C., C. Cabalin y V. Orellana, The 2011 Chilean Student Movement Against Neoliberal Educational Policies, doi: 10.1080/03075079.2014.896179, Studies in Higher Education, 39(3), 426-440 (2014)

Brint, S., A. Cantwell y R. Hanneman, The Two Cultures of Undergraduate Academic Engagement, doi: 10.1007/s11162008-9090-y, Research in Higher Education, 49(5), 383-402 (2008)

Carey, P., The Impact of Institutional Culture, Policy and Process on Student Engagement in University Decision-Making, doi: 10.1080/13603108.2016.1168754, Perspectives: Policy and Practice in Higher Education, 22(1), 11-18 (2016)

Carini, R., G. Kuh y S. Klein, Student Engagement and Student Learning: Testing the Linkages, doi: 10.1007/s11162-0058150-9, Research in Higher Education, 47(1), 1-32 (2006)

Chen, P. D., T.N. Ingram y L.K. Davis, Bridging Student Engagement and Satisfaction: A Comparison between Historically Black Colleges and Universities and Predominantly White Institutions, doi: 10.7709/jnegroeducation.83.4.0565, Journal of Negro Education, 83(4), 565-579 (2014)

Coates, H., The Value of Student Engagement for Higher Education Quality Assurance, doi: 10.1080/13538320500074915, Quality in Higher Education, 11(1), 25-36 (2005)

Denson, N. y M. Chang, Racial Diversity Matters: The Impact Of Diversity-Related Student Engagement and Institutional Context, doi: 10.3102/0002831208323278, American Educational Research Journal, 46(2), $322-353$ (2008)

Díaz, C., Modelo Conceptual para la Deserción Estudiantil Universitaria Chilena, doi: 10.4067/S071807052008000200004, Estudios Pedagógicos, 34(2), 65-86 (2008)

Egerton, M., Higher Education and Civic Engagement, doi: 10.1080/0007131022000021506, British Journal of Sociology, 53(4), 603-620 (2002)

González, J., C. Carvajal y F. Viveros, Coeficientes Edumétricos para la Validez y Dificultad de un Test: Propuesta, doi: 10.4067/S0718-07052016000400025, Estudios Pedagógicos, 42(3), 467-481 (2016)

$\mathrm{Hu}$, S. y A. McCormick, An Engagement-Based Student Typology and Its Relationship to College Outcomes, doi: 10.1007/s11162-012-9254-7, Research in Higher Education, 53(7), 738-754 (2012)

Hu, S. y G.C. Wolniak, College Student Engagement and Early Career Earnings: Differences By Gender, Race/Ethnicity, And Academic Preparation, doi: 10.1353/rhe.2013.0002, The Review of Higher Education, 36(2), 211-233 (2013)

Jackling, B. y R. Natoli, Student Engagement and Departure Intention: An Australian University Perspective, doi: 10.1080/0309877X.2011.584970, Journal of Further and Higher Education, 35(4), 561-579 (2011) 
Jacoby, B., Civic Engagement in Today's Higher Education: An Overview, en B. Jacoby y T. Ehrlich (Eds.), Civic Engagement in higher education. Concept and practices, pp. 5-30, Jossey Bass, San Francisco, EE.UU. (2009)

Jelas, Z.M., A. Azman, H. Zulnaidi y N.A. Ahmad, Learning Support and Academic Achievement among Malaysian Adolescents: The Mediating Role Of Student Engagement, doi: 10.1007/s10984-015-9202-5, Learning Environments Research, 19(2), 221-240 (2016)

Kahn, P. E., Theorising Student Engagement in Higher Education, doi: 10.1002/berj.3121, British Educational Research Journal, 40(6), 1005-1018 (2014)

Kahn, P.E., Higher Education Policy on Student Engagement: Thinking Outside the Box, doi: 10.1057/s41307-016-00317, Higher Education Policy, 30(1), 53-68 (2017)

Kahu, E.R., Framing Student Engagement in Higher Education, doi: 10.1080/03075079.2011.598505, Studies in Higher Education, 38(5), 758-773 (2013)

Kahu, E.R. y K. Nelson, Student Engagement in The Educational Interface: Understanding the Mechanisms of Student Success, doi: 10.1080/07294360.2017.1344197, Higher Education Research and Development, 37(1), 58-71 (2017)

Krause, K. y H. Coates, Students' Engagement in First-Year University, doi: 10.1080/02602930701698892, Assessment and Evaluation in Higher Education, 33(5), 493-505 (2008)

Krause, K., Addressing the Wicked Problem of Quality in Higher Education: Theoretical Approaches and Implications, doi: 10.1080/07294360.2011.634381, Higher Education Research and Development, 31(3), 285-297 (2012)

Kuh, G., T. Cruce y otros tres autores, Unmasking the Effects of Student Engagement on First-Year College Grades and Persistence, doi: 10.1353/jhe.0.0019, The Journal of Higher Education, 79(5), 540-563 (2008)

Kuh, G., The National Survey of Student Engagement: Conceptual and Empirical Foundations, doi: 10.1002/ir.283, New Directions for Institutional Research, 2009(141), 5-20 (2009)

LaNasa, S., A. Cabrera y H. Trangsrud, The Construct Validity of Student Engagement: A Confirmatory Factor Analysis Approach, doi: 10.1007/s11162-009-9123-1, Research in Higher Education, 50(3), 315-332 (2009)

Lester, J., J. Brown-Leonard y D. Mathias, Transfer Student Engagement: Blurring of Social and Academic Engagement, doi: 10.1177/0091552113496141, Community College Review, 41(3), 202-222 (2013)

McMahon, B. y J. Portelli, Engagement for What? Beyond Popular Discourses of Student Engagement, doi: 10.1076/lpos.3.1.59.27841, Leadership and Policy in Schools, 3(1), 59-76 (2004)

McMahon, B. y J. Portelli, The Challenges of Neoliberalism in Education: Implications for Student Engagement, in B. McMahon y J. Portelli (Eds.), Student Engagement in Urban School: Beyond Neoliberal Discourses, pp. 1-10, Information Age Publishing, Charlotte - NC, EE.UU. (2012)

Namakforoosh, M., Metodología de la Investigación, $2^{\text {da }}$ Ed., 209-218, Limusa, México D. F., México (2005)

National Survey of Student Engagement (NSSE), Topical Module: Civic Engagement, https://goo.gl/ycHu51, 12-16-14[v2], Indiana University, EE.UU. (2016)

National Survey of Student Engagement (NSSE), Survey Instrument, https://goo.gl/W8To5L, Indiana University, EE.UU. (2017)

Palominos, F. E., H.M. Díaz, S.K .Palominos y L.R. Cañete, Relación entre los Procedimientos de Selección a la Educación Superior y el Desempeño Académico de los Estudiantes con base en una Clasificación mediante Conjuntos Difusos, doi: https://dx.doi.org/10.4067/S0718-50062018000100045, Formación Universitaria, 11(1), 45-52 (2018)

Pascarella, E., T. Seifert y C. Blaich, How Effective Are the NSSE Benchmarks in Predicting Important Educational Outcomes? doi: 10.1080/00091380903449060, Change: The Magazine of Higher Learning, 42(1), 16-22 (2010)

Pineda-Báez, C., J. Bermúdez-Aponte y otros cuatro autores, Compromiso Estudiantil y Desempeño Académico en el Contexto Universitario Colombiano, doi: 10.7203/relieve.20.2.4238, RELIEVE, 20(2), 1-20 (2014)

Valenzuela, M. J., K. Romero, C. Vidal-Silva y A. Philominraj, Factores que Influyen en el Aprendizaje del Idioma Inglés de Nivel Inicial en una Universidad Chilena, doi: https://dx.doi.org/10.4067/S0718-50062016000600006, Formación Universitaria, 9(6), 63-72 (2016)

Wimpenny, K. y M. Savin-Baden, Alienation, Agency and Authenticity: A Synthesis of the Literature on Student Engagement, doi: 10.1080/13562517.2012.725223, Teaching in Higher Education, 18(3), 311-326 (2013)

Zepke, N. y L. Leach, Beyond Hard Outcomes: Soft Outcomes and Engagement as Student Success, doi: 10.1080/13562517.2010.522084, Teaching in Higher Education, 15(6), 661-673 (2010)

Zepke, N., Student Engagement Research in Higher Education: Questioning an Academic Orthodoxy, doi: 10.1080/13562517.2014.901956, Teaching in Higher Education, 19(6), 697-708 (2014)

Zepke, N., Student Engagement and Neoliberalism: Mapping an Elective Affinity, doi: 10.1080/02601370.2015.1096312, International Journal of Lifelong Education, 34(6), 696-709 (2015)

Zepke, N., Student Engagement in Neoliberal Times, 95-130, Springer, Singapore (2017) 
